\title{
Unusual causes of peritonitis in a peritoneal dialysis patient: Alcaligenes faecalis and Pantoea agglomerans
}

\author{
Arzu Kahveci ${ }^{* *}$, Ebru Asicioglu', Elif Tigen ${ }^{2}$, Elif Ari ${ }^{1}$, Hakki Arikan', Zekaver Odabasi ${ }^{2}$ and Cetin Ozener ${ }^{1}$
}

\begin{abstract}
An 87 -year-old female who was undergoing peritoneal dialysis presented with peritonitis caused by Alcaligenes faecalis and Pantoea agglomerans in consecutive years. With the following report we discuss the importance of these unusual microorganisms in peritoneal dialysis patients.
\end{abstract}

\section{Case report}

The most common pathogens causing peritonitis in peritoneal dialysis (PD) patients are gram positive organisms, Staphylococcus epidermidis and Staphylococcus aureus. Gram negative organisms and fungi are less common causes. Environmental organisms are rarely implicated in patients with peritonitis. With the following report we describe two peritonitis episodes in successive years caused by two unusual microorganism: Alcaligenes faecalis and Pantoea agglomerans.

A 89-year-old female patient receiving automated peritoneal dialysis presented with abdominal pain and cloudy dialysis fluid. She had a history of diabetes mellitus and had been maintained on APD because of diabetic nephropathy for 10 years. In 2004 she had culture negative peritonitis which was treated with sefazoline and ciprofloxacin. In January 2007, she presented with abdominal pain, cloudy dialysate, and vomiting. Analysis of peritoneal effluent demonstrated white blood cell (WBC) of $700 / \mathrm{mm}^{3}$. Fifty $\mathrm{mL}$ of peritoneal fluid was centrifugated at $3000 \mathrm{~g}$ for 15 minutes, then resuspension of the sediment in 3-5 mL of steril salin inoculated in chocolate-agar, blood-agar and MacConkey agar in microbiology unit. Intraperitoneal sefuroksim $(750 \mathrm{mg}$ $\mathrm{q} 12 \mathrm{~h}$ ) and oral ciprofloxacin (500 mg q12h) were given empirically. On the fifth day of treatment a gram-nega-

\footnotetext{
* Correspondence: drarzukahveci@yahoo.com

${ }^{1}$ Marmara University School of Medicine, Department of Internal Medicine, Division of Nephrology, Istanbul, Turkey

Full list of author information is available at the end of the article
}

tive, oxidase-positive bacteria with peritrichous flagella, Alcaligenes faecalis was isolated from MacConkey agar. The antibiotic treatment was continued for 21 days and she recovered completely. On February 2009, she presented with cloudy dialysate and abdominal pain. On physical examination, blood pressure was $60 / 40 \mathrm{mmHg}$, pulse rate was $96 / \mathrm{min}$, body temperature was $36.5^{\circ} \mathrm{C}$. Cardiopulmonary examination was normal. Her abdomen was distended and tender, with normal bowel sounds. Laboratory data showed a blood WBC of $14000 / \mathrm{mm}^{3}$. Analysis of peritoneal fluid demonstrated WBC $2700 / \mathrm{mm}^{3}$. She was treated with intraperitoneal sefuroksim (750 mg q12 h) and ciprofloxacin (200 mg q12 h) empirically after peritoneal fluid culture sampling. On third day of treatment peritoneal fluid WBC increased to $3400 / \mathrm{mm}^{3}$, abdominal pain persisted and the dialysis fluid cultures grew Gram negative rods for which imipenem was started. On blood and chocolate agar plates, the gram-negative rods displayed yellow pigmentation (Figure 1). Oxidase was negative and catalase was positive. The strain was identified as Pantoea agglomerans. The patient's history was not remarkable regarding the possible plant associated injury. On the sixth day, she refused to undergo removal of her PD catheter. Even though appropriate antibiotic therapy was administrated, she died due to septic shock.

\section{Discussion}

Alcaligenes faecalis and Pantoea agglomerans are classified as environmental bacteria and plant pathogens and 


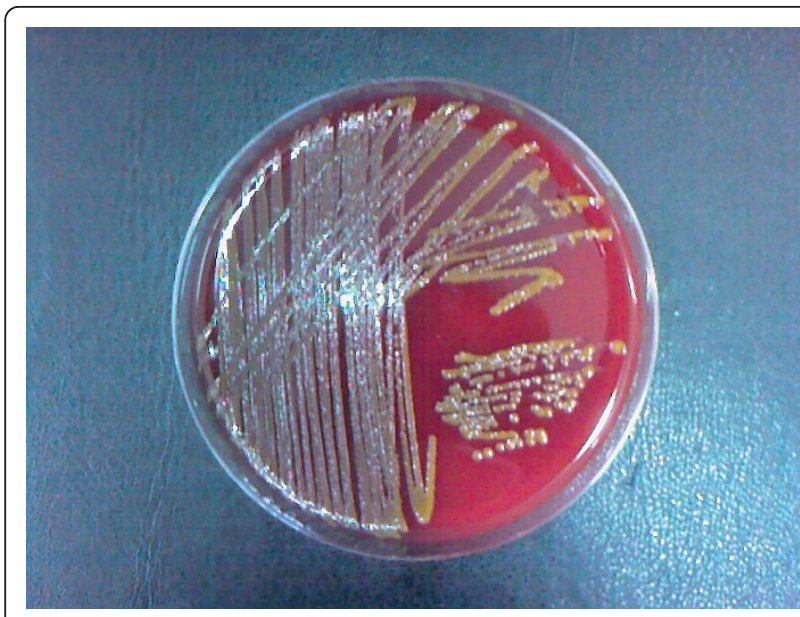

Figure 1 Pantoea agglomerans strain which produces yellow pigmentation on blood agar.

are rarely reported as being responsible for clinically significant infections in human. Alcaligenes faecalis is a gram negative oxidase positive rod with peritrichous flagella which exists in soil and water. It is also found in the alimentary tract as a harmless saprophyte in 5-19\% of the human population. Systemic infection with this organism is very uncommon. To our knowledge, this is the second report of a PD patient with Alcaligenes faecalis peritonitis. This patient recovered by antimicrobial therapy alone and did not require catheter removal similar to previous patient reported by Kavuncuoglu et al. [1]. However another species of Alcaligenes, Alcaligenes xylosoxidans has been reported to cause peritonitis in PD patients previously [2]. The majority of these patients required catheter removal because of resistance to most antibiotic therapies. Even though Alcaligenes faecalis is presumed to be resistant to antibiotics, as in our isolate, our case emphasizes the importance of sensitivity testing when it is isolated.
Pantoea agglomerans is another gram negative aerobic bacillus which caused peritonitis in our patient in 2009. It can be isolated from plants, soil and feculent material. Pantoea species are rare causes of clinically significant infection similar to A. Faecalis. However in recent years, an increased rate of Pantoea peritonitis in PD patients has been reported (Table 1) [3-9]. Plant associated injury is the most commonly attributed aetiology. However it has been linked to contaminated blood products, intravenous fluids, total parenteral nutrition and anesthetic agents. Furthermore oral contamination or bacterial translocation from gastrointestinal system may also be responsible for Pantoea infections. Peritoneal dialysis catheters are not known to be a risk factor of infection by Pantoea [5]. Regarding the previous Alcaligenes faecalis peritonitis in our patient we believe that plant associated injury or bacterial translocation may be responsible factors for the peritonitis episodes.

In conclusion, although these organisms are rarely fatal and are commonly considered to be contaminants they can cause symptomatic peritonitis, which in turn leads to death. It is therefore very important to view these organisms as pathogens rather than contaminants when isolated in patients with peritonitis. Microbiological antibiotic testing should always be requested from the laboratory and patients should be treated accordingly. When there is no improvement with antibiotic therapy, early removal of the peritoneal catheter should be planned since these infections may prove fatal.

\section{Consent}

Written informed consent was obtained from the patient's daughter for publication of this case report and accompanying images. A copy of the written consent is available for review by the Editor-in-Chief of this journal.

Table 1 Cases of Peritoneal Dialysis (PD) Associated Peritonitis with Pantoae Agglomerans

\begin{tabular}{llllll}
\hline Case (Ref.) & Type of PD & Sex/Age & Proposed etiology & Antbiotics used/route & Outcome \\
\hline $1[3]$ & NA & F/2 & Teeting to catheter & Cefotaxime, Gentamisin/IP & Cured, catheter replaced new one \\
\hline $2[4]$ & CAPD & F/49 & Rose-thorn injury & Ceftazidime, Amikasin/IP & Cured \\
\hline $3[5]$ & CCPD & M/65 & Unknown & Ciprofloxacin/IV & Cured \\
\hline $4[6]$ & CAPD & $M / 51$ & Rose-thorn injury & Cefepime/IP & Cured \\
\hline $5[7]$ & NA & F/52 & Unknown & Ciprofloxacin/oral & Cured \\
\hline $6[8]$ & CAPD & $\mathrm{M} / 45$ & Thorn injury & Ciprofloxacin/IP & Cured \\
\hline $7[9]$ & CCPD & $M / 56$ & Unknown & Tobramycin/NA & Cured \\
\hline Our case & APD & F/89 & Unknown & İmipenem/IV & Patient died \\
\hline
\end{tabular}

NA, not adjusted; CAPD, Continuous Ambulatory Peritoneal Dialysis; CCPD, Continuous Cycling Peritoneal Daialysis; APD, Automated Peritoneal Dialysis; IP, Intraperitoneal; IV, Intravenous. 


\section{Author details}

'Marmara University School of Medicine, Department of Internal Medicine, Division of Nephrology, Istanbul, Turkey. ${ }^{2}$ Marmara University School of Medicine, Department of Infectious Diseases, Istanbul, Turkey.

\section{Authors' contributions}

$A K, E A, E T$ and EA made substantial contributions to the design, and the acquisition and interpretation of data. $\mathrm{HA}, \mathrm{ZO}$ and $\mathrm{CO}$ revised the manuscript critically for important intellectual content. All authors read and approved the final manuscript.

\section{Competing interests}

The authors declare that they have no competing interests.

Received: 24 January 2011 Accepted: 10 April 2011

Published: 10 April 2011

\section{References}

1. Kavuncuoglu F, Unal A, Oguzhan N, Tokgoz B, Oymak O, Utas C: First Reported Case of Alcaligenes faecalis Peritonitis. Perit Dial Int 2010, 30(1):118-119.

2. Ledger SG, Cordy P: Successful treatment of Alcaligenes xylosoxidans in automated peritoneal dialysis-related peritonitis. Perit Dial Int 2007, 27(5):596-598.

3. Lau KK, Ault BH, Jones DP: Polymicrobial peritonitis including Pantoea agglomerans from teething on a catheter. South Med J 2005, 98(5):580-581.

4. Lim PS, Chen SL, Tsai CY, Pai MA: Pantoea peritonitis in a patient receiving chronic ambulatory peritoneal dialysis. Nephrology (Carlton) 2006, 11(2):97-99.

5. Magnette C, Tintillier M, Horlait G, Cuvelier C, Pochet JM: Severe peritonitis due to Pantoea agglomerans in a CCPD patient. Perit Dial Int 2008, 28(2):207-208

6. Ferrantino $M$, Navaneethan SD, Sloand JA: Pantoea agglomerans: an unusual inciting agent in peritonitis. Perit Dial Int 2008, 28(4):428-430.

7. Habhab W, Blake PG: Pantoea peritonitis: not just a "thorny" problem. Perit Dial Int 2008, 28(4):430.

8. Moreiras-Plaza M, Blanco-García R, Romero-Jung P, Feijóo-Piñeiro D, Fernandez-Fernandez C, Ammari I: Pantoea agglomerans: the gardener's peritonitis? Clin Nephrol 72(2):159-161.

9. Borràs M, Roig J, Garcia M, Fernández E: Adverse effects of pantoea peritonitis on peritoneal transport. Perit Dial Int 2009, 29(2):234-235.

\section{doi:10.1186/1476-0711-10-12}

Cite this article as: Kahveci et al:: Unusual causes of peritonitis in a peritoneal dialysis patient: Alcaligenes faecalis and Pantoea agglomerans. Annals of Clinical Microbiology and Antimicrobials 2011 10:12.

\section{Submit your next manuscript to BioMed Central and take full advantage of:}

- Convenient online submission

- Thorough peer review

- No space constraints or color figure charges

- Immediate publication on acceptance

- Inclusion in PubMed, CAS, Scopus and Google Scholar

- Research which is freely available for redistribution 